\title{
Application of Electronic Chart Display and Information System in the Teaching of Nautical English
}

\author{
Xiandong Zhao ${ }^{\mathrm{a}}$, Yonggang Zong ${ }^{\mathrm{b}}$ \\ Binzhou Polytechnic, No.919, Huanghe 12 Road, Binzhou, Shandong Province, China. \\ a315722535@qq.com, b510115338@qq.com,
}

Keywords: Electronic chart display and information system; nautical English; teaching; system design.

\begin{abstract}
The emergence of Electronic Chart Display and Information System (ECDIS) is a revolution in the field of navigation. With the improvement of ECDIS's standardization and its further promotion, the research of ECDIS in the world is also deepening. On the basis of the understanding of the standard of electronic chart, many countries have applied ECDIS to many fields and made remarkable achievements. The use of ECDIS in the teaching of nautical English can further improve the function of the teaching of nautical English. China's nautical English teaching project is mostly based on ECDIS to realize its management function.
\end{abstract}

\section{Introduction}

The International Maritime Organization (IMO) requires ships of ports and international navigation in all countries to use English as a communication tool for working language. On the one hand, the difficulties, misunderstandings and obstacles of English communication are serious threats to the safety of the ship. On the other hand, Chinese sailors had a serious relative surplus. The relative surplus is due to the low quality of seafarers, especially the low comprehensive ability of nautical English. Nautical English has become the biggest obstacle for Chinese crew go to the world, which has seriously restricted the development of higher nautical education and vocational crew training in China. The existing nautical English teaching and training mode already cannot adapt to the requirements of modern navigation for personnel training. It is urgent how to use modern educational technology like network teaching to conduct the corresponding reform and development of teaching system and teaching methods of nautical English [1].

\section{Theory}

\subsection{Standard of Electronic Chart}

At present, the electronic chart system has developed from the early simple navigation system display terminal to the current multi-functional electronic chart display and information system ECDIS. The emergence of ECDIS is a revolution in the field of navigation. It plays a more and more important role in ensuring the safety of navigation and strengthening the management of ship traffic [2]. In order to make this system able to embark on an international unified track, the International Hydrographic Organization (IHO) and the International Maritime Organization (IMO) as well as the International Commission of Electrotechnical (ICE) have jointly formulated the ECDIS performance, display and data standards. These standards include: IOH S-57, namely digital hydrographic data transmission standard: IHO S-52, namely the ECDIS chart content and display specification: IMO ECDIS performance standard; IEC61174, namely ECDIS hardware performance and test standard [3].

\subsection{Definition of Electronic Chart}

Based on the introduction to these criteria, we can understand ECDIS in this way: to meet the requirements of IMO ECDIS performance, the chart information used should be the latest information conforming to IHO standard provided by the official Hydrographic Department. ECDIS should be able to receive formal correction data conforming to IHO standard, and the IHO standard is 
IHO S-57 [4]. What stored in the database is electronic chart in the vector form. The description of chart information is based on the classification and coding system format given in S-57. The data structure adopts S-57 format, while the data record is wrapped in ISO/IEC8211 format when transmitting and exchanging. The specific content, display mode, color and symbol usage of electronic chart and so on should conform to the IHO S-52 specification. The hardware device of ECDIS must be tested through IEC performance test [5].

\subsection{Data Format of Electronic Chart}

The research on the ECDIS display is based on the data storage method of the electronic chart as the premise, which is the data source of the ECDIS. The following is an introduction to the data storage and exchange format of the standard electronic chart, S-57. The S-57 worker ISO/IEC 8211:1994 is used as its data encapsulation standard. This standard stipulates eight aspects: data structure, coding general protocol, meta record coding protocol, feature record and coding agreement, space record and coding convention, relation coding, structure application and correction. The S-57 format of the electronic chart can be divided into two aspects, the logical structure and the physical structure [6].

\subsection{Logic Structure of S-57}

In the logical structure, the entity in the real world is described by the combination of the feature object and the space object. The characteristic objects include meta objects, cartographic objects, geographical objects and set objects. They describe the attributes, properties and characteristics of objects. Spatial objects include nodes, sides and planes. They describe the spatial location characteristics of entities [7].

$\mathrm{D}$ in the surface; the surface is contained in isolated nodes: the edge is the boundary of the surface; the boundary is the boundary on the surface; the end point of the edge is the associated node; the destination of the edge is the related node; and the edge is adjacent to the surface. The feature object is located by one or more spatial objects. The feature object can also do not contain the corresponding spatial objects, but each space object must have the characteristic object for the reference [8]. There can also be a mutual reference relationship between the feature objects to indicate the interrelation between them. The above analysis is as shown in Figure 1.

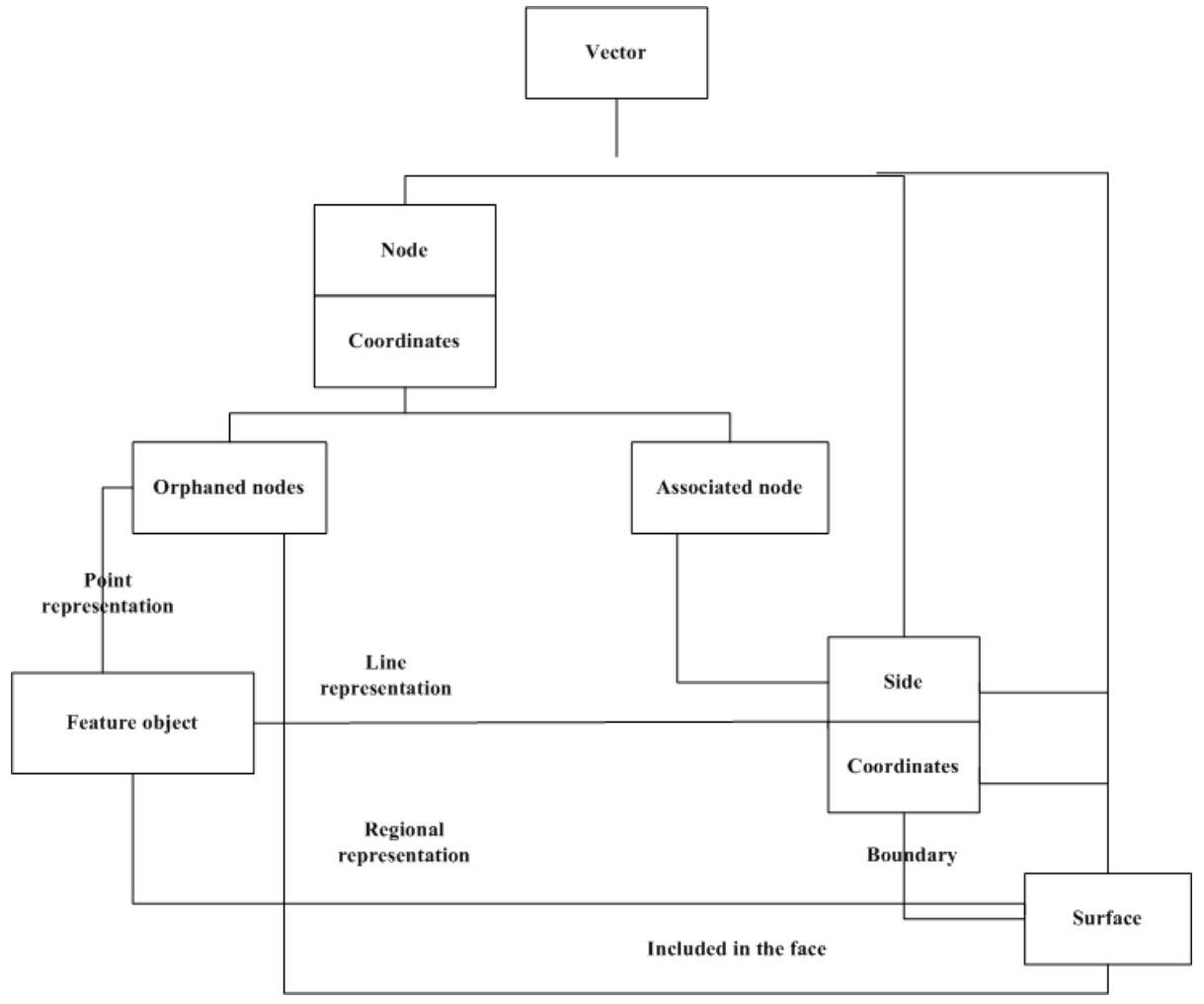

Figure 1 Chart vector topological structure diagram 


\section{Method}

\subsection{Design of Electronic Chart Display System}

The display of electronic chart is a very mature technology. As a subsystem of ECDIS, considering the mathematical basis and algorithm, it mainly includes three parts: the synthesis of electronic chart, the presentation of the electronic chart after synthesis and the electronic chart editor. The composition structure of the electronic chart display system is shown in Figure 2.

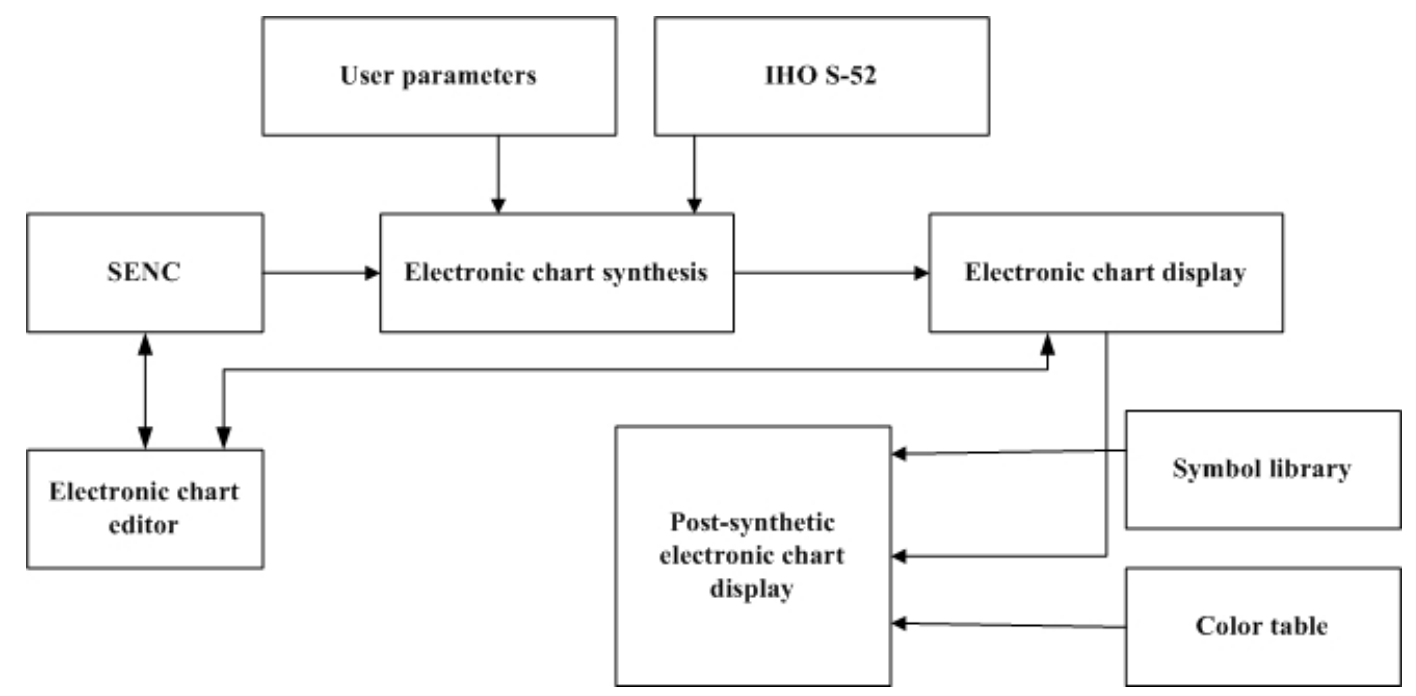

Figure 2 The composition structure of the electronic chart display system

\subsection{Realization of The Symbol Library of the Electronic Chart By Using Supermap III}

In the above display process, a structural unit contained is a symbol library. The S-52 standard has relevant specifications for the symbols of the electronic chart and gives a standard symbol library. However, this symbol library only has some fixed symbols. And in application, in many cases, especially in nautical English teaching, it requiring users to design some symbols. As a result, it needs to design a symbol editor module, and these two parts are contained in the symbol library as mentioned above. Because the electronic chart is a special geographic information system (GIS), in this electronic chart display, we use the GIS development tool SuperMap III to design and implement the symbol library of the electronic chart.

Today's popular geographic information system mainly uses the form of symbol library to manage map symbols, and realizes the production of map symbols with the idea of interactive design. The symbol library design approach of GIS relevant software at home and abroad mainly has four kinds. The first is a text editor design method and construction of a symbol text description. The second is using the development programming language provided by the system for the implementation, which is mainly used in rendering of special symbols. The third is the use of the system's graphic editing function to regard the symbols as ordinary graphics block design and additional symbol description code stored in the symbol library. The fourth is to provide the symbol design interface. The user can combine the basic symbol provided by the system and customized simple symbol, and after displacement and zoom, the required complex symbols output by the drawings can be obtained (such as linear complex symbols and areal symbols), which is currently popular symbol design method.

\subsection{Design of Symbolic Function Module}

The realization of symbolic function module is, according to correspondence between object code established in coded control table file and symbols in the symbol library, to draw and output the spatial entity in the mapping output range with the specified symbols (i.e., symbol), with its as the center. The symbolic process is shown in Figure 3. 


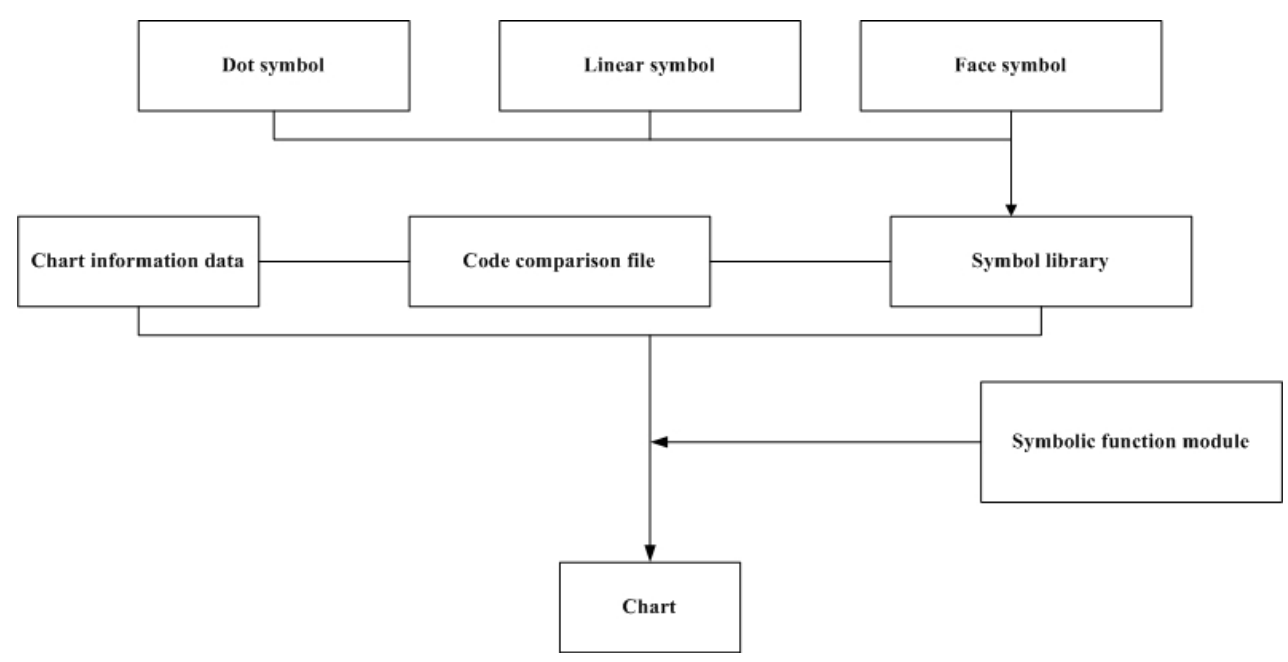

Figure 3 Symbolic function module

The detailed steps of the above process are as follows:

The geometric coordinates and attribute parameters of the symbols to be symbolized are obtained from the geographic database according to the mapping output range; according to the object code in the attribute parameter table, in the coded control table file, the symbol code and the symbol to display the color and other parameters are found when the object marks are symbolized; the symbol information of the symbol code is obtained from the symbol library; the symbolic function module symbolized the object standard according to the information described by the geometric position and symbol of the object mark, and output the symbolic result.

In this process, before the symbolic processing, it is necessary to do a preliminary review of geographic data, such as if boundary coordinate string of plane target is closed. In the symbolic processing, symbols' color, style, and size are determined by the symbol attribute characteristics. It is necessary to judge and calculate symbolic computation parameter setting after extracting data from the relevant attribute table. After the symbolic processing, further treatment should be done, such as burr remove (in building topological relation and solving arc intersection, it often produces small short-term goals), the target line hanging point discard and so on.

\section{Implementation}

\subsection{Contents Realized By the System}

VHF communication: "Calling at Singapore” ship (captain) - harbor; "Joining the Convoy” ship harbor.

Diversion of water into port: "Pilot Comes On Board" and "Berthing” captain

Someone comes on board: "Agent Comes On Board”; "Harbour Officer Comes On Board”; "Foreman Comes On Board”; "Before Discharging”; "During Discharging”; “On Completion Of Discharging”; "Storage Plan”; "Before Loading”; "During Loading”; “On Completion Of Loading”

\subsection{Interface Setting}

According to the above requirements, after logical induction and summary, the user interaction interface is identified as the following seven parts based on the principles of clarity, conciseness and simplicity.

Role playing: when the user presses this key, the system gives a window on which there are various roles that the user can play and the ON/OFF switch. There are "determine" and "give up" two buttons, and it returns to the main interface through them; setting: when the user presses the button, the system gives a window on which there are "auto play" and "manual play", "Chinese tips" and "English tips" button and the speed adjustment slider. There are "determine" and "give up" two buttons, and it returns to the main interface through them; playing: when the user has set up the required functions and the key is pressed, the dialog system starts to run; pausing: when it is in a playback state, we press this button and the dialog system operation pauses; stopping: whenever this 
key is pressed, the system returns to the initial state; exit: Whenever this key is pressed, the system jumps to the main caller; page control: It is made up of "forward" and "backward" two buttons, so as to complete the function that should be responsible for.

\subsection{Major Implemented Functions of the Dictionary Section}

Search queries: when a user clicks a letter on the title bar, the word that begins with the letter will be displayed in the database; carry out fuzzy query of words. But when users are not clear about spelling of a word, it can use part of the characters of the word to query, and the query result will display the word containing the string; screen words selection: make use of wordclickde system function in authorware, to capture words; add the word: it is very convenient to add the words, and if the word already exists, it cannot be added.

\subsection{Realization of the Test Question System}

First of all, in the lead-in section, the computer explains to students the purpose and requirement of exercise and self-test, and introduces the dynamic management and commonly used operation methods of courseware. If students choose the exercise system, the system shows the type and level of the courseware contained exercises, for students to choose. When students choose the computer, it enters into a cycle: choose exercises, display exercises, ask the students to answer, judge the answers and provide feedback information to students. This cycle is repeated so that the cycle is withdrawn until a certain condition is met. The conditions are selected by the students, and can also be controlled by the program. Finally, the program enters the end section. If students choose the self-lateral test system, a test paper will be displayed according to certain rules for students to turn over to test. After the answer is finished, students can count their scores and get the corresponding feedback information. The feedback information will influence the next set of rules. If the student enters the system as a manager, it can dynamically update and adjust the press and problem rules, as shown in Figure 4.

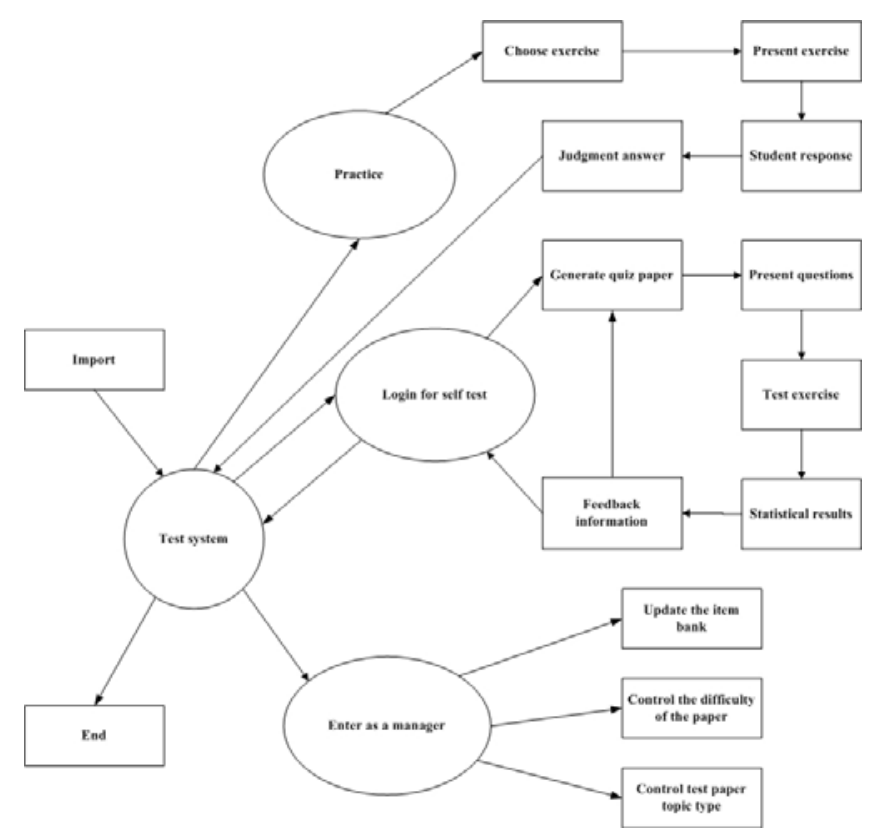

Figure 4 The logical basic structure of the exercise system

\subsection{Achievement of Dialogue Part Database Structure}

The dialogue part database is mainly for the convenience of the management of text, and the revision of the text content, especially the management of the voice file. The specific database structure is as follows:

\begin{tabular}{|c|c|c|}
\hline Field name & Attributes & Whether it is the keyword \\
\hline EnglishS & Note & No \\
\hline ChinesesE & Note & No \\
\hline DialogNo & Number & Yes \\
\hline SoundFile & Note & No \\
\hline
\end{tabular}


Eglishs (English sentences) and ChinesesE (Chinese explanation) use the node type: because the string length in English sentence and Chinese explanation data is very large, the data type text type cannot meet the requirements, we choose the note type.

DialogNo (dialogue number): The digital key is used and defined as the primary key, because the user can jump to a sentence at any time when he uses the dialogue part, and it is easy to find for the database after it is defined as the primary key.

SoundFile (sound file): we use note type to store the file name of voice files and then use the system function Filelocation to locate the files. During playback, we assign the location of files to the playback icon in variable form, which can play the voice files.

In addition, because the dialogue part is the training with course as the unit and the user can select any lesson to practice, we use the course number as table name. Moreover, in that each lesson is divided into different parts, it also needs to add a suffix. For example: if the table name is 1a, it suggests a part of the first lesson. In this way, when the user selects a part of a lesson, it not only can open the corresponding database, but also read out the data.

\subsection{Function Realization of Dialogue Part}

The dialogue part, first of all, provides users with a course selection interface. When users click, they will enter the corresponding courses, choose the curriculum part that they want to train, and then enter into the scene for training. The automatic configuration icons in the mainstream map are also used to complete the automatic configuration of the ODBC database, convenient for the procedures to be packaged. The source code is as follows:

DB Retype: $=4$

DB Type: =” Mierosoft Aeeess Driver (*.mdb) "

DB List: =”DSN=dict:"

DB List: =DB List”Description=ODBC DSN:"

DB List: =DB List”Aeeess:”

DB List: =DBList”DBQ="Filelocation” dialogic database.mdb:"

OCDB Driverlnstalled: =tMsDBRegister (DB ReqType. DB Type. DB List).

After the automatic configuration is finished, the flow chart enters the course selection section. When the user selects a lesson, it calls the corresponding program.

\section{Conclusion}

The courseware makes full use of multimedia characteristics and organically integrates sound, image, picture and text and animation. By means of scene simulation, dialogue observation, man-machine practice, fun vocabulary memorizing, instant dictionary, random testing and so on ways, we break through the plane limit of books and teaching materials and the single linear model of audio-visual reading. As a result, we make dull English learning interesting, improve the students' initiative and enthusiasm, and fully mobilize the various senses of human access to information. It enhances students' understanding and improves memory and learning efficiency, thus greatly improving the students' English listening and speaking training level and comprehensively improving students' practical ability of nautical English.

\section{References}

[1]. IBRAHIM, A. M.; TAWFIK, AHMED KHALIL. Educational technology in MET simulator based training and information technology in MET. Int J Mech Eng, 2015, 4.3: 1-10.

[2]. CRESSWELL, Kathrin M., et al. Workarounds to hospital electronic prescribing systems: a qualitative study in English hospitals. BMJ Qual Saf, 2017, 26.7: 542-551.

[3]. ZHANG, Wenjun; XIAO, Zhongming; LIU, Jiyong. A Study on Curriculum of Practical Teaching in Nautical Science. Journal of Residuals Science \& Technology, 2016, 13.8. 
[4]. HYMAN, Jack A.; MOSER, Mary T.; SEGALA, Laura N. Electronic reading and digital library technologies: Understanding learner expectation and usage intent for mobile learning. Educational Technology Research and Development, 2014, 62.1: 35-52.

[5]. HYMAN, Jack A.; MOSER, Mary T.; SEGALA, Laura N. Electronic reading and digital library technologies: Understanding learner expectation and usage intent for mobile learning. Educational Technology Research and Development, 2014, 62.1: 35-52.

[6]. FUNG, Doric. PC connectable electronic learning aid device with replaceable activity worksheets. U.S. Patent No 8,655,258, 2014.

[7]. SHAFFER, Alexandra V.; SANTAMARIA, Jonathan B. Electronic system, method and device operable to facilitate education. U.S. Patent Application No 14/699,593, 2015.

[8]. DUKHON, Marina, et al. Exposing non-authoring features through document status information in an out-space user interface. U.S. Patent No 8,762,880, 2014. 